\title{
Infections in urology: slow progress reflected in clinical practice
}

\author{
Selcuk Guven ${ }^{1} \cdot$ Ali Mert $^{2}$ \\ Published online: 6 October 2020 \\ (c) Springer-Verlag GmbH Germany, part of Springer Nature 2020
}

This special topic issue on infections in urology features works from scientists, infectious disease specialists, and urologists. As seen in this work, there is still a noticeable heterogeneity in the global burden of the disease [1]. Indeed, while deficiencies in health systems and documentation restrict our epidemiological knowledge, the disease's epidemiology is changing with globalization and increasing migration events. Infections such as tuberculosis, brucellosis, schistosomiasis, filariasis, and hydatidosis as well as infectious diseases associated with multidrug resistance (MDR), extensively drug-resistance (XDR), and pan drugresistance (PDR) generally follow geographic distribution patterns with the help of human travel and trade. These diseases pose a new challenge for doctors who need to diagnose conditions that have not been seen before. They also make it challenging to address new drug-resistant organisms, which reveal that these diseases, which are thought to have been controlled, should be revisited [2-4].

The ongoing challenge of unnecessary treatments has grown to be a limitation. Overtreatment of asymptomatic candiduria with antifungals is frequently seen in daily clinical practice. The Candida score or colonization index is used to distinguish Candida colonization and invasive candidiasis in non-neutropenic critical patients. The presence of Candida colonization at multiple body sites (i.e., urine, respiratory secretions, oral, rectal, and inguinal) may be an important clue for treatment [5]. Specifically, such scoring systems gain particular importance in the frail elderly population because of a lack of evidence-based guidelines. Urinary tract infections (UTIs) are the leading causes of

Selcuk Guven

selcukguven@hotmail.com

Ali Mert

alimert@medipol.edu.tr

1 Department of Urology, Meram Medical Faculty, Necmettin Erbakan University, Konya, Turkey

2 Department of Infectious Diseases and Clinical Microbiology, Istanbul Medipol University, Istanbul, Turkey bacteremia and require empiric antimicrobial therapy and hospitalization. Decreased functional status, urosepsis, or even death may occur in the frail elderly population [6]. Significant advances in basic science and in genomic studies have been made to understand the disease and the infection agent. With continued evolution of next-generation molecular technologies, there is now hope that these new techniques may predict the probability of severe infection development and the microbiome of the patient in an asymptomatic population. Based on the likelihood of these predictions, action can be taken to design targeted antibiotic management. This would indeed represent progress for individualized patient care [7].

As profiles of microbiomes and viromes of the bladder are beginning to surface, it is possible to better understand the contribution of other members of the kingdom of complex urinary system diseases [8]. With the introduction of bacterial gene testing technology, the number of bacterial strains and rates that could not be achieved in traditional bacterial culture technology may now be clarified. The role of catheters, which are frequently used in urinary obstructions and infections associated with MDR, is known. The bacterial gene test can provide information about the diversity of bacterial flora in the urine of patients with urinary catheterization $[9,10]$. However, it is hard to say that these advances are reflected in clinical practice. While our expectations for new generation molecular diagnosis methods and treatments are growing, limitations on the burden and epidemiology of the disease continue. As the challenges and constraints related to the disease and its causal agent have continued, surgical solutions for the disease have developed. The contributions of industry and engineering technology in surgical solutions and the interest of endourologists in this matter have encouraged the use of minimally invasive interventions. Many obstructive infections that have previously relied on surgeries and eventually progressed to disease and kidney failure can now be dealt with through minimally invasive interventions. Repeated interventions due to chronic complications of infection can be eliminated or even postponed without damaging the patient's anatomy. 
Inherently, every new treatment modality brings some drawbacks. For example, sepsis may develop in percutaneous stone interventions, and health care professionals try to eliminate or reduce complications related to these procedures [11]. In addition, during the urgent management of acute obstruction and sepsis, providers in all health care settings can apply culture samples from percutaneous nephrostomy to deliver improvements in antimicrobial stewardship [12].

In Aydin et al.'s articles on renal transplantation and urinary infections, they addressed the issues through the following five key topics: incidences, risk factors, current treatment practices, and controversial topics [13]. Derin et al. pointed to future antibiotic resistance in the prostate biopsy and recommended the use of rectal swab culture and nucleic acid amplification tests (NAATs). Tailoring a patient's antimicrobial prophylaxis based on rectal swab culture may increase the effectiveness of antimicrobial prophylaxis. NAATs, which are used to detect fluoroquinolone resistance, may also identify all antibiotic-resistant rectal microbiota members, including MDRs [14].

Besides the new sophisticated technologies, preventive measures play a useful role in reducing the rate of health care-associated urinary tract infection (HAUTI) and in optimizing their management. HAUTI is a major concern, considering its prevalence of 7.7\% (4.6-17.3\%) in urology departments, a rate that is higher than other departments $(0.5-1.7 \%)$. Previously, Tandogdu et al. illustrated that antibiotic choices could be different between HAUTI conditions. Analysis of the data utilizing the Bayesian weighted incidence syndromic antibiogram (WISCA) approach, to a certain extent, unmasked previously unknown findings [15]. These findings can improve empirical antibiotic selection towards a personalized approach. An active program for the control of HAUTI must be in place, and this program must include medical staff, nurses, patients, and patients' relatives. The study also demonstrated that observational assessment might reduce catheter-associated infection, with rates falling from $9.1 \%$ in 2012 to $5.2 \%$ in 2018 [16].

Another uncertainty in clinical practice is who is going to diagnose the patient and who will provide the follow-up or treatment. Although there is no clear answer to this issue in the literature, a urologist might be the moderator of this chronic process. Since the urologist is the physician who most frequently faces this condition in daily practice, they are most aware of the uroanatomy as well as the pathophysiology and can perform relevant interventions when needed. At this point, rather than limiting the evaluation and treatment to a single specialty, it is crucial to solve the problem using a multidisciplinary approach [17].

\section{References}

1. Öztürk R, Murt A (2020) Epidemiology of urological infections: a global burden. World J Urol. https://doi.org/10.1007/s00345-01903071-4

2. Batirel A, Regmi SK, Singh P et al (2020) Urological infections in the developing world: an increasing problem in developed countries. World J Urol. https://doi.org/10.1007/s00345-020-03120-3

3. Mert A, Guzelburc V, Guven S (2020) Urinary tuberculosis: still a challenge. World J Urol. https://doi.org/10.1007/s00345-02003146-7

4. Kulchavenya E, Kholtobin D, Shevchenko S (2020) Challenges in urogenital tuberculosis. World J Urol 38:89-94. https://doi. org/10.1007/s00345-019-02767-x

5. Odabasi Z, Mert A (2019) Candida urinary tract infections in adults. World J Urol. https://doi.org/10.1007/s00345-019-02991 $-5$

6. Zeng G, Zhu W, Lam W et al (2020) Treatment of urinary tract infections in the old and fragile. World J Urol. https://doi. org/10.1007/s00345-020-03159-2

7. Dixon M, Stefil M, McDonald M et al (2020) Metagenomics in diagnosis and improved targeted treatment of UTI. World J Urol 38:35-43. https://doi.org/10.1007/s00345-019-02731-9

8. Yıldırım S, Shoskes D, Kulkarni S et al (2020) Urinary microbiome in uncomplicated and interstitial cystitis: is there any similarity? World J Urol. https://doi.org/10.1007/s00345-020-03099-x

9. Li X, Gao H, Sun X et al (2020) A preliminary study on the role of Bacteroidesfragilis in stent encrustation. World J Urol. https:// doi.org/10.1007/s00345-020-03185-0

10. Wang S, Zhang Y, Zhang X et al (2020) An evaluation of multidrug-resistant (MDR) bacteria in patients with urinary stone disease: data from a high-volume stone management center. World $\mathbf{J}$ Urol 38:425-432. https://doi.org/10.1007/s00345-019-02772-0

11. Zhao Z, Wu W, Zeng T et al (2020) The impact of nephrostomy drainage prior to mini-percutaneous nephrolithotomy in patients with ESBL-positive Escherichia coli. World J Urol. https://doi. org/10.1007/s00345-020-03155-6

12. Batura D, Gopal Rao G (2020) A systematic review of the clinical significance of nephrostomy urine cultures. World J Urol 38:4555. https://doi.org/10.1007/s00345-019-02663-4

13. Aydın S, Patil A, Desai M et al (2020) Five compelling UTI questions after kidney transplant. World J Urol. https://doi. org/10.1007/s00345-020-03173-4

14. Derin O, Fonseca L, Sanchez-Salas R et al (2020) Infectious complications of prostate biopsy: winning battles but not war. World J Urol. https://doi.org/10.1007/s00345-020-03112-3

15. Tandogdu Z, Koves B, Cai T et al (2020) Condition-specific surveillance in health care-associated urinary tract infections as a strategy to improve empirical antibiotic treatment: an epidemiological modelling study. World J Urol 38:27-34. https://doi. org/10.1007/s00345-019-02963-9

16. Medina-Polo J, Gil-Moradillo J, Justo-Quintas J et al (2020) Prevention of healthcare-associated infections (HAIs) in a surgical urology ward: observational study-analysis of the problem and strategies for implementation. World J Urol 38:3-8. https://doi. org/10.1007/s00345-019-02648-3

17. Soytas M, Kactan C, Guven S (2020) Recurrent bladder cystitis: who takes the role? World J Urol. https://doi.org/10.1007/s0034 5-020-03192-1

Publisher's Note Springer Nature remains neutral with regard to jurisdictional claims in published maps and institutional affiliations. 